\title{
Is the Random Forest Algorithm Suitable for Predicting Parkinson's Disease with Mild Cognitive Impairment out of Parkinson's Disease with Normal Cognition?
}

\author{
Haewon Byeon $(1)$ \\ Department of Speech Language Pathology, School of Public Health, Honam University, Gwangju 62399, Korea; \\ bhwpuma@naver.com
}

Received: 6 March 2020; Accepted: 7 April 2020; Published: 10 April 2020

\begin{abstract}
Because it is possible to delay the progression of dementia if it is detected and treated in an early stage, identifying mild cognitive impairment (MCI) is an important primary goal of dementia treatment. The objectives of this study were to develop a random forest-based Parkinson's disease with mild cognitive impairment (PD-MCI) prediction model considering health behaviors, environmental factors, medical history, physical functions, depression, and cognitive functions using the Parkinson's Dementia Clinical Epidemiology Data (a national survey conducted by the Korea Centers for Disease Control and Prevention) and to compare the prediction accuracy of our model with those of decision tree and multiple logistic regression models. We analyzed 96 subjects (PD-MCI $=45$; Parkinson's disease with normal cognition (PD-NC) $=51$ subjects). The prediction accuracy of the model was calculated using the overall accuracy, sensitivity, and specificity. Based on the random forest analysis, the major risk factors of PD-MCI were, in descending order of magnitude, Clinical Dementia Rating (CDR) sum of boxes, Untitled Parkinson's Disease Rating (UPDRS) motor score, the Korean Mini Mental State Examination (K-MMSE) total score, and the K- Korean Montreal Cognitive Assessment (K-MoCA) total score. The random forest method achieved a higher sensitivity than the decision tree model. Thus, it is advisable to develop a protocol to easily identify early stage PDD based on the PD-MCI prediction model developed in this study, in order to establish individualized monitoring to track high-risk groups.
\end{abstract}

Keywords: cognitive function; data mining; Parkinson's disease with mild cognitive impairment; random forest; neuropsychological test

\section{Introduction}

Over the past decade, the field of geriatrics has experienced emerging interest in Parkinson's disease with mild cognitive impairment (PD-MCI) [1-4]. The Sydney cohort study [5], the most highly representative epidemiology study on the subject, examined 136 patients diagnosed with Parkinson's disease (PD) over 20 years. The study reported that $84 \%$ of PD patients had cognitive impairment, and $50 \%$ of them progressed to PD dementia (PDD). Likewise, PD is often accompanied by cognitive dysfunction in addition to dyskinesia [2].

The mild cognitive impairment (MCI) stage is the earliest at which we can detect dementia [6]. Because it is possible to delay the progression of dementia when it is detected and treated in an early stage, identifying MCI is an important primary goal of dementia treatment [6]. PD-MCI is frequently found in patients with $\mathrm{PD}[7,8]$. However, the sociodemographic and neuropsychological characteristics of PD-MCI are less well-known than those of $\mathrm{MCI}$ and vascular mild cognitive impairment (vascular-MCI) [7,8]. The distinctive neuropsychological characteristics found in early stage 
PD-MCI are caused by executive function damage due to prefrontal hypofunction or malfunction [9]. However, it is difficult to distinguish PD-MCI from MCI or vascular-MCI, because they show similar symptoms [10]. Additionally, people with PD experience a slowly deteriorating cognitive deficit and impaired motor function, which can be mistaken for cognitive frailty as part of the normal aging process. As a result, it is difficult to diagnose early stage PD. MCI can be diagnosed based on interviewing, cognitive function evaluation via a standardized neuropsychological test, and brain imaging such as magnetic resonance imaging (MRI). It is possible to diagnose cerebrovascular diseases or to analyze brain atrophy using brain imaging. However, this is unsuitable for early PD diagnosis, because brain atrophy can be confirmed visually only at a very advanced stage. Therefore, neuropsychological testing that also tests cognitive function has been used as an effective screening test for diagnosing MCI [11].

Recent studies have pointed to the necessity of considering mental health, such as depression, while diagnosing MCI $[12,13]$. In particular, the development pattern and risk factors of cognitive impairment are known to vary according to race. Therefore, it is necessary to develop an MCI prediction model reflecting the characteristics of the neuropsychological indices and lifestyles of the elderly in South Korea; however, South Korea has less systematic epidemiological data on cognitive impairment in the elderly than other countries such as the United States and European countries. In South Korea, previous community-based epidemiological studies on PD have been conducted on patients living in a single city [14]. However, there has been no study to develop a prediction model based on a nationwide epidemiological survey. Moreover, most of the previous studies $[15,16]$ evaluating the neuropsychological characteristics of patients with PD have used regression models. Regression models are effective in exploring the neuropsychological characteristics of individual risk factors but are limited in analyzing multiple risk factors simultaneously. It is also difficult to prioritize risk factors with regression models. Linear regression models in particular require several assumptions, including linearity, equal variance, and a normal distribution, but disease data have been known to violate these assumptions.

In recent years, the medical field has applied data mining to predict the risk of diseases and vulnerable groups [16,17]. Data mining is a type of big data analysis that examines the relationships and rules within a dataset to extract valuable information [18]. The health science field has traditionally used tree-based methods such as Classification and Regression Tree (CART) as data mining methods for disease prediction [19]. Decision trees carry the risk of overfitting, and the accuracy of decision trees can vary greatly depending on the training data (input variables). Random forests, a data mining method developed in 2001, were designed to overcome these limitations. Random forests generate multiple decision trees by conducting random sampling on the same dataset and combining them to predict the target variable. Therefore, the accuracy of random forests is higher than that of decision trees $[20,21]$. Moreover, random forests can be used to explore the relationship between explanatory variables and diseases when many (types of) explanatory variables are applied to a random forest model [22]. In addition, the prediction power of random forests outperforms the bagging model [22].

Several previous studies [23-25] have reported on Parkinson's dementia predictors using biomarkers such as cerebrospinal fluid (CSF) and electroencephalogram (EEG) data. However, we are unaware of any study that identifies the predictors of PD-MCI for patients with PD and normal cognition (PD-NC), taking into account sociodemographic factors, lifestyles, depression, and neuropsychological characteristics. The objectives of this study were to develop a random forest-based PD-MCI prediction model considering health behaviors, environmental factors, medical history, physical functions, depression, and cognitive functions by using the Parkinson's Dementia Clinical Epidemiology Data (a national survey conducted by the Korea Centers for Disease Control and Prevention), and to compare its prediction of accuracy with those of decision tree and multiple logistic regression models. 


\section{Methods}

\subsection{Data Source}

This study was conducted using the Parkinson's Dementia Clinical Epidemiology Data obtained from the National Biobank of Korea, the Center for Disease Control and Prevention, the Republic of Korea (no. KBN-2019-005). We obtained the approval of the Research Ethics Review Board, the National Biobank of Korea (no. KBN-2019-005), and the data use approval of the Korea Centers for Disease Control and Prevention (no. KBN-2019-1327). The National Biobank of Korea was established in 2008 with the approval of the Ministry of Health and Welfare and is managed by the Korea Centers for Disease Control and Prevention for the emerging necessity of managing bio-data systematically at a national level. The ultimate goal of the National Biobank of Korea is to promote biomedical research and public health. Please refer to Lee et al. [26] for the specific activities of the National Biobank of Korea, including its quality control programs.

The Parkinson's Dementia Clinical Epidemiology Data used in this study were collected under the supervision of the Korea Centers for Disease Control and Prevention at 14 tertiary care organizations (university hospitals) from January to December 2015. Health surveys, including health behavior questions, were conducted using computer-assisted personal interviews. The data are composed of sociodemographic factors (e.g., gender), environmental factors (e.g., exposure to pesticides), health behaviors (e.g., smoking), disease history (e.g., hypertension), exercise characteristics related to PD (e.g., tremor), sleep behavior disorders (e.g., rapid eye movement (REM)), and neuropsychological characteristics (e.g., cognitive function). PD-MCI was diagnosed by neuropsychologists according to the criteria of the International Working Group on MCI [27].

\subsection{Subjects}

Observational studies frequently utilize secondary data and these studies are more likely to experience data imbalance while comparing patients and healthy subjects [28]. Propensity score matching (PSM) was used to minimize selection bias and resolve the imbalance of case-control [29]. This study found an imbalance between PD-NC and PD-MCI. In order to solve this issue, this study used PSM, balancing between populations using the nearest neighbor matching by controlling the age of the case-control group [30]. Moreover, this study excluded individuals (subjects) that did not match in both groups in common to ensure good data balance. Before matching, there were 274 subjects $(\mathrm{PD}-\mathrm{MCI}=223$; PD-NC = 51), and, after conducting PSM, it was matched to 96 subjects (PD-MCI = 45, PD-NC $=51$; Figure 1). This study finally analyzed 96 subjects. 


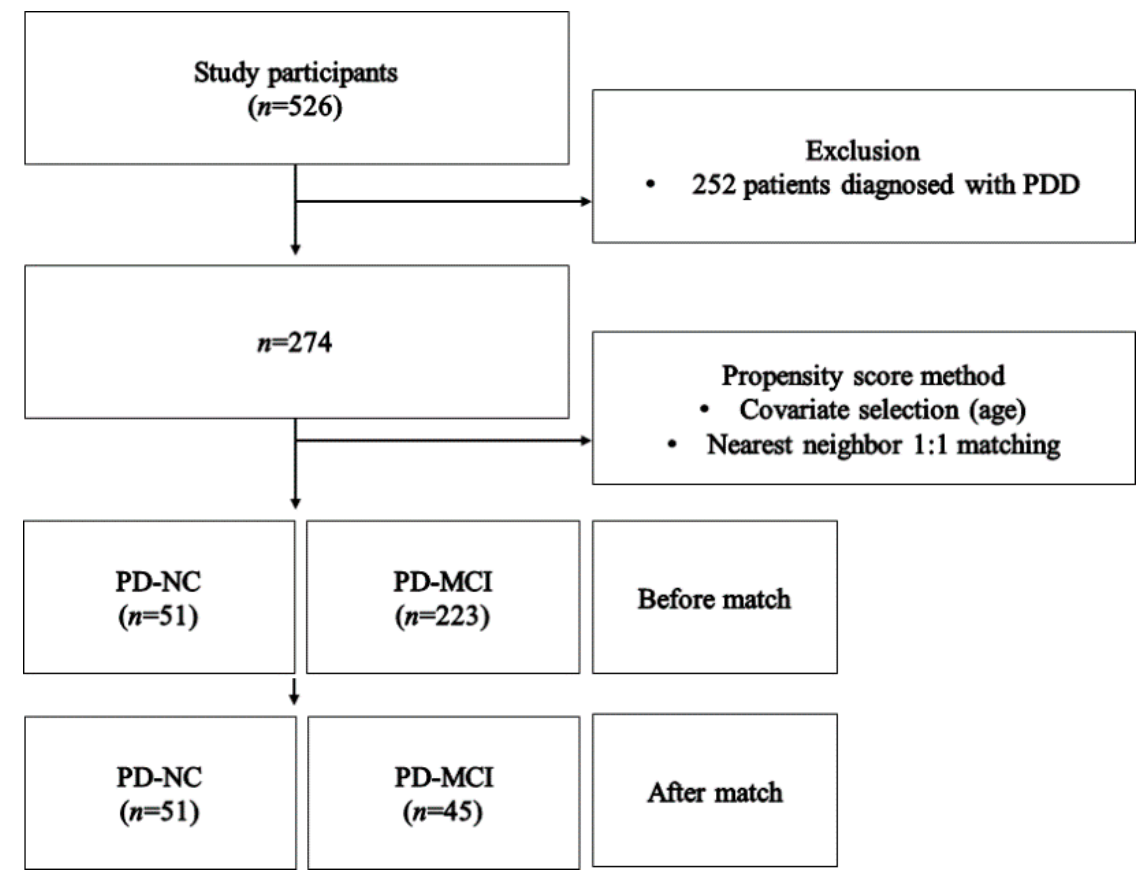

Figure 1. Framework of study.

\subsection{Measurement}

The outcome variable is defined as the prevalence of PD-MCI classified by medical diagnosis. The explanatory variables included age (60-74 years old or $\geq 75$ years old), gender (male or female), education (middle school graduate and below, or high school graduate and above), handedness (left hand, right hand, or both hands), family dementia history (yes or no), family PD history (yes or no), pack-years (non-smoking, 1-20, 21-40, 41-60, or $\geq 61$ pack-years), coffee-drinking (yes or no), mean coffee intake per day (no, $\leq 1,2-3$, or $\geq 4$ cups), coffee drinking period (no, $\leq 5,6-9$, or $\geq 10$ years), pesticide exposure (never, currently not exposed but exposed previously, or currently exposed to pesticide), disease history (carbon monoxide poisoning, manganese poisoning, encephalitis, traumatic brain injury, stroke, alcoholism, diabetes, hypertension, hyperlipidemia, and/or atrial fibrillation), PD related motor signs (tremor, akinesia/bradykinesia, postural instability, and/or late motor complications), REM, sleep behavior disorders, neuropsychological characteristics such as those outlined in the Korean Mini Mental State Examination (K-MMSE) [31], Korean Montreal Cognitive Assessment (K-MoCA) [32], Geriatric Depression Score (GDS) [33], global Clinical Dementia Rating (CDR) score [34], Korean Instrumental Activities of Daily Living (K-IADL) score [35], Untitled Parkinson's Disease Rating (UPDRS) total score [36], UPDRS motor score [37], Hoehn and Yahr staging (H\&Y staging) [38], and the Schwab and England Activities of Daily Living scale (Schwab and England ADL) [39]. These variables are defined in Table 1. 
Table 1. Measurement and definition of variables.

\begin{tabular}{|c|c|c|}
\hline Variable. & Measurement & Characteristics \\
\hline \multirow{6}{*}{ Sociodemographic factors } & Gender & Male or female \\
\hline & Education & $\begin{array}{l}\text { Middle school graduate and below or high school } \\
\text { graduate and above }\end{array}$ \\
\hline & Mainly used hand & Left hand, right hand, or both hands \\
\hline & Family dementia history & Yes or no \\
\hline & Family PD history & Yes or no \\
\hline & Pack-years & Non-smoking, $1-20,21-40$, or $\geq 41$ pack-years \\
\hline \multirow{4}{*}{ Health behaviors } & Coffee-drinking & Yes or no \\
\hline & Mean coffee intake per day (cups/day) & No, $\leq 1,2-3$, or $\geq 4$ cups \\
\hline & Coffee drinking period (year) & No, $\leq 5,6-9$, or $\geq 10$ years \\
\hline & Exposure to pesticide & $\begin{array}{l}\text { Never, currently not exposed but exposed previously, } \\
\text { or currently exposed to pesticide }\end{array}$ \\
\hline Environmental factors & Carbon monoxide poisoning & Yes or no \\
\hline \multirow{8}{*}{ Disease history } & Manganese poisoning & Yes or no \\
\hline & Traumatic brain injury & Yes or no \\
\hline & Stroke & Yes or no \\
\hline & Diabetes & Yes or no \\
\hline & Hypertension & Yes or no \\
\hline & Hyperlipidemia & Yes or no \\
\hline & Atrial fibrillation & Yes or no \\
\hline & Tremor & Yes or no \\
\hline \multirow{4}{*}{$\begin{array}{l}\text { Exercise characteristics related to } \\
\text { PD (PD related motor signs) }\end{array}$} & Rigidity & Yes or no \\
\hline & Bradykinesia & Yes or no \\
\hline & Postural instability & Yes or no \\
\hline & $\begin{array}{l}\text { Rapid eye movement (REM) and sleep } \\
\text { behavior disorders (RBD) }\end{array}$ & Yes or no \\
\hline Sleep behavior disorders & Total score of K-MMSE & Continuous variable \\
\hline Neuropsychological characteristics & $\begin{array}{l}\text { Total score of K-MoCA } \\
\text { CDR global score } \\
\text { CDR sum of boxes } \\
\text { K-IADL } \\
\text { Total score of UPDRS } \\
\text { Motor score of UPDRS } \\
\text { H\&Y staging (Hoehn and Yahr staging) } \\
\text { Schwab and England ADL }\end{array}$ & Continuous variable \\
\hline
\end{tabular}

Pack-years: Cumulative amount of smoking, based on one pack of smoking per day. For example, 30 pack-years means smoking one pack of cigarettes per day for 30 years or two packs of cigarettes per day for 15 years. CDR-Clinical Dementia Rating; K-IADL-Korean Instrumental Activities of Daily Living; UDPRS-Untitled Parkinson's Disease Rating; ADL-Schwab and England Activities of Daily Living scale.

\subsection{Development and Evaluation of Prediction Models}

The prediction model was developed using a random forest algorithm, and the results of the developed prediction model were compared with those of a decision tree based on multiple logistic regression and a classification and regression tree. The prediction accuracy of the model was calculated using the recognition rate.

Random forests are ensemble classifiers that randomly learn multiple decision trees. The random forest method consists of a training step that constructs several decision trees, and a test step that classifies or predicts an outcome variable based on an input vector. The ensemble form of random forest training data can be expressed as Forest $F=\{\mathrm{f} 1, \ldots, \mathrm{f} n\}$ (Figure 2). The distributions obtained from the decision trees of each forest were first averaged by $\mathrm{T}$ (the number of the decision trees) and then classification was conducted. The predictors of each sample were combined by using the mean for continuous target variables and the majority vote for categorical target variables.

$$
L(p)=\frac{1}{T} \sum_{t=1}^{T} P_{t}(b \mid I, p)
$$




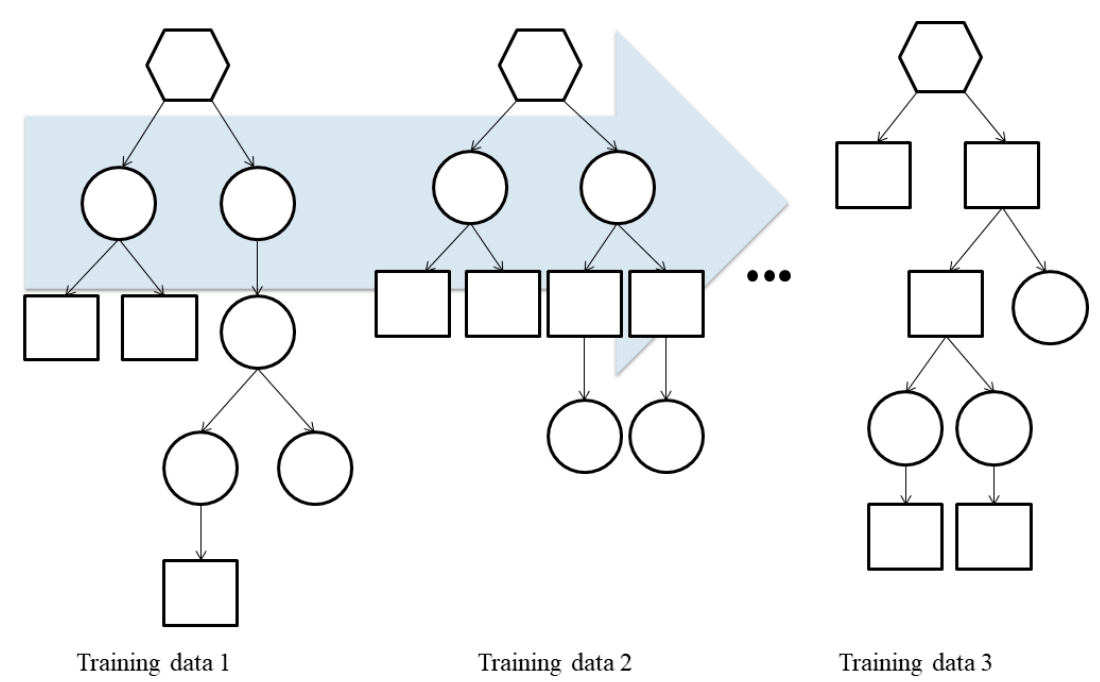

Figure 2. Ensemble classifiers that combines many single decision trees.

Random forest is similar to the bagging technique, because both approaches combine decision trees generated from multiple bootstrap samples using the majority vote principle in order to increase stability. However, they are different, because the former uses a few explanatory variables that were randomly selected from each bootstrap sample.

This study presented a partial dependence plot and variable importance to show the prediction power of the main explanatory variables. The variable importance indicates the effect of an explanatory variable on the accuracy of a model. Therefore, when an explanatory variable improves the performance of a model, the importance of the variable increases. A partial dependence plot shows the changes in response variables according to the continuous change of each explanatory variable. The contribution of a dependent variable to an independent variable is expressed as a function of a variable. The function of partial dependence is presented in Equation (2).

$$
\left(\frac{p_{1}\left(x, x_{i c}\right)}{p_{0}\left(x, x_{i c}\right)}\right)
$$

RF can be free from overfitting theoretically, and is not affected by noise or outliers much [20]. Moreover, it can generate high accuracy results by reducing generalization errors [20]. However, RF is more likely to have an elbow point, which means a steep drop in slope with more trees. Moreover, there is a higher probability that each tree will be more complex when an unimportant explanatory variable is selected. Therefore, this study improved the accuracy of the model by considering the number of mtry, the number of candidate explanatory variables, in advance.

The prediction performance of a model was validated while considering the overall accuracy, sensitivity, and specificity together. Sensitivity means the prediction accuracy of PD-MCI, while specificity indicates that of PD-NC. As the objective of this study was to develop a model that can predict PD-MCI, this study considered overall prediction accuracy and sensitivity as the most important factors for evaluating prediction performance. When the overall prediction accuracies and sensitivities of the two models were identical, their specificities were compared. This study first established a random forest model and then compared the results and the accuracies of models obtained from multiple logistic regression and CART. In this case, forward selection based on standard likelihood ratio tests was used to select variables in the multiple logistic regression analysis. All of the statistical analyses were conducted using the "RandomForest" package of R-version-3.6.1 (Foundation for Statistical Computing, Vienna, Austria). 


\section{Results}

\subsection{General Characteristics of the Subjects}

The General characteristics of the subjects are presented in Table 2. Of the 96 subjects (after match), $47.9 \%$ were male, $52.1 \%$ were female, $38.5 \%$ had a high school or above level of education, $8.0 \%$ had a family history of PD, and 6.8\% had a family history of Alzheimer's dementia. Additionally, 5.7\%, $2.3 \%, 23.2 \%$, and $40.0 \%$ of the subjects had a history of head injury (e.g., traumatic brain injury), stroke, diabetes, and hypertension, respectively.

Table 2. General characteristics of the subjects, $n(\%)$.

\begin{tabular}{|c|c|c|c|}
\hline \multirow{2}{*}{ Characteristics } & \multicolumn{3}{|c|}{ After Match } \\
\hline & PD-MCI $(n=45)$ & PD-NC $(n=51)$ & Total $(n=96)$ \\
\hline \multicolumn{4}{|l|}{ Gender } \\
\hline Male & $24(53.3)$ & $22(43.1)$ & $46(47.9)$ \\
\hline Female & $21(46.7)$ & $29(56.9)$ & $50(52.1)$ \\
\hline \multicolumn{4}{|l|}{ Education } \\
\hline $\begin{array}{l}\text { Middle school } \\
\text { graduate and below }\end{array}$ & $27(60.0)$ & $32(62.7)$ & $59(61.5)$ \\
\hline $\begin{array}{l}\text { High school } \\
\text { graduate and above }\end{array}$ & $18(40.0)$ & $19(37.3)$ & $37(38.5)$ \\
\hline \multicolumn{4}{|l|}{ Mainly used hand } \\
\hline Right hand & $44(97.8)$ & $47(92.2)$ & $91(94.8)$ \\
\hline Left hand & $1(2.2)$ & $1(2.0)$ & $2(2.1)$ \\
\hline Both hands & 0 & $3(5.9)$ & $3(3.1)$ \\
\hline \multicolumn{4}{|l|}{ Family PD history } \\
\hline No & $36(92.3)$ & $33(91.7)$ & $69(92.0)$ \\
\hline Yes & $3(7.7)$ & $3(8.3)$ & $6(8.0)$ \\
\hline \multicolumn{4}{|c|}{ Family dementia history } \\
\hline No & $36(94.7)$ & $32(91.4)$ & $68(93.2)$ \\
\hline Yes & $2(5.3)$ & $3(8.6)$ & $5(6.8)$ \\
\hline \multicolumn{4}{|l|}{ Pack year (Smoking) } \\
\hline $1-20$ & $6(13.3)$ & $3(5.9)$ & $9(9.4)$ \\
\hline $21-40$ & $3(6.7)$ & $2(3.9)$ & $5(5.2)$ \\
\hline $41+$ & $36(80.0)$ & $46(90.2)$ & $82(85.4)$ \\
\hline \multicolumn{4}{|l|}{ Coffee-drinking } \\
\hline No & $15(33.3)$ & $19(37.3)$ & $34(35.4)$ \\
\hline Yes & $30(66.7)$ & $32(62.7)$ & $57(64.6)$ \\
\hline \multicolumn{4}{|l|}{$\begin{array}{l}\text { Carbon monoxide } \\
\text { poisoning }\end{array}$} \\
\hline No & $42(97.7)$ & $38(86.4)$ & $80(92.0)$ \\
\hline Yes & $1(2.3)$ & 6 (13.6) & $7(8.0)$ \\
\hline \multicolumn{4}{|l|}{ Traumatic brain injury } \\
\hline No & $40(93.0)$ & $42(95.5)$ & $82(94.3)$ \\
\hline Yes & $3(7.0)$ & $2(4.5)$ & $5(5.7)$ \\
\hline \multicolumn{4}{|l|}{ Stroke } \\
\hline No & $41(95.3)$ & $44(100)$ & $85(97.7)$ \\
\hline Yes & $2(4.7)$ & 0 & $2(2.3)$ \\
\hline \multicolumn{4}{|l|}{ Diabetes } \\
\hline No & $36(80.0)$ & $37(74.4)$ & $73(76.8)$ \\
\hline Yes & $9(20.0)$ & $13(26.0)$ & $22(23.2)$ \\
\hline \multicolumn{4}{|l|}{ Hypertension } \\
\hline No & $32(71.1)$ & $25(50.0)$ & $57(60.0)$ \\
\hline Yes & $13(28.9)$ & $25(50.0)$ & $38(40.0)$ \\
\hline \multicolumn{4}{|l|}{ Hyperlipidemia } \\
\hline No & $41(91.1)$ & $43(86.0)$ & $84(88.4)$ \\
\hline Yes & $4(8.9)$ & $7(14.0)$ & $11(11.6)$ \\
\hline \multicolumn{4}{|l|}{ Atrial fibrillation } \\
\hline No & $44(97.8)$ & $47(94.0)$ & $91(95.8)$ \\
\hline Yes & $1(2.2)$ & $3(6.0)$ & $4(4.2)$ \\
\hline \multicolumn{4}{|l|}{ Tremor } \\
\hline No & $14(33.3)$ & $8(17.4)$ & $22(25.0)$ \\
\hline Yes & $28(66.7)$ & $38(82.6)$ & $66(75.0)$ \\
\hline
\end{tabular}


Table 2. Cont.

\begin{tabular}{|c|c|c|c|}
\hline \multirow{2}{*}{ Characteristics } & \multicolumn{3}{|c|}{ After Match } \\
\hline & PD-MCI $(n=45)$ & PD-NC $(n=51)$ & Total $(n=96)$ \\
\hline \multicolumn{4}{|l|}{ Rigidity } \\
\hline No & $3(7.0)$ & $8(17.0)$ & $11(12.2)$ \\
\hline Yes & 40 (93.0) & $39(83.0)$ & 79 (87.8) \\
\hline \multicolumn{4}{|l|}{ Bradykinesia } \\
\hline No & $2(4.7)$ & $6(12.8)$ & $8(8.9)$ \\
\hline Yes & $41(95.3)$ & $41(87.2)$ & $82(91.1)$ \\
\hline \multicolumn{4}{|l|}{ Postural instability } \\
\hline No & $22(55.0)$ & $28(60.9)$ & $50(58.1)$ \\
\hline Yes & $18(45.0)$ & $18(39.1)$ & $36(41.9)$ \\
\hline \multicolumn{4}{|l|}{$\begin{array}{l}\text { REM sleep behavior } \\
\text { disorders }\end{array}$} \\
\hline No & $29(67.4)$ & $27(56.3)$ & $56(61.5)$ \\
\hline Yes & $14(32.6)$ & $21(43.7)$ & 35 (38.5) \\
\hline \multicolumn{4}{|l|}{ Depression (GDS) } \\
\hline No & $22(62.9)$ & $22(75.9)$ & $44(68.8)$ \\
\hline Yes & $13(37.1)$ & $7(24.1)$ & $20(31.3)$ \\
\hline K-MMSE, mean \pm SD & $25.8 \pm 2.7$ & $25.4 \pm 4.7$ & $25.6 \pm 3.9$ \\
\hline K-MoCA, mean \pm SD & $20.6 \pm 4.0$ & $20.5 \pm 6.2$ & $20.5 \pm 5.3$ \\
\hline $\begin{array}{l}\text { Global CDR score, mean } \\
\pm \text { SD }\end{array}$ & $0.5 \pm 0.2$ & $0.5 \pm 0.6$ & $0.5 \pm 0.4$ \\
\hline $\begin{array}{l}\text { Sum of boxes in CDR, } \\
\text { mean } \pm \text { SD }\end{array}$ & $1.4 \pm 1.4$ & $0.8 \pm 1.3$ & $1.2 \pm 1.4$ \\
\hline K-IADL, mean \pm SD & $1.0 \pm 2.6$ & $0.7 \pm 1.0$ & $0.8 \pm 2.0$ \\
\hline Total UPDRS, mean \pm SD & $34.9 \pm 18.9$ & $29.9 \pm 13.1$ & $33.0 \pm 16.9$ \\
\hline $\begin{array}{l}\text { Motor UPDRS, mean } \pm \\
\text { SD }\end{array}$ & $22.6 \pm 11.6$ & $17.9 \pm 8.6$ & $20.0 \pm 10.3$ \\
\hline $\begin{array}{l}\text { H\&Y staging score, mean } \\
\pm \mathrm{SD}\end{array}$ & $2.1 \pm 0.8$ & $1.8 \pm 0.6$ & $2.0 \pm 0.7$ \\
\hline $\begin{array}{l}\text { Schwab and England } \\
\text { ADL, mean } \pm \text { SD }\end{array}$ & $80.0 \pm 16.0$ & $87.7 \pm 8.1$ & $83.6 \pm 13.3$ \\
\hline
\end{tabular}

REM sleep behavior disorders—rapid eye movement sleep behavior disorders; PD-MCI—Parkinson's Disease with Mild Cognitive Impairment; PD-NC—Parkinson's Disease with Normal Cognition; K-MMSE-Korean Mini Mental State Examination; K-MoCA—Korean Montreal Cognitive Assessment; CDR—Clinical Dementia Rating; K-IADL-Korean Instrumental Activities of Daily Living; UPDRS—Untitled Parkinson's Disease Rating; H\&Y staging — Hoehn and Yahr staging; Schwab and England ADL—Schwab and England Activities of Daily Living scale.

\subsection{Major Risk Factors of Random Forest-Based PD-MCI Prediction Model}

A PD-MCI prediction model was established using random forests, and the results are presented in Figure 3. Some of the random forest models estimated major risk factors using decreased in the GINI coefficient. The major risk factors of PD-MCI were, in descending order of magnitude, CDR sum of boxes, UPDRS motor score, the K-MMSE total score, and the K-MoCA total score. Among these factors, the UPDRS motor score was the most important predictor of PD-MCI. In contrast, the importance of atrial fibrillation and stroke was zero. 


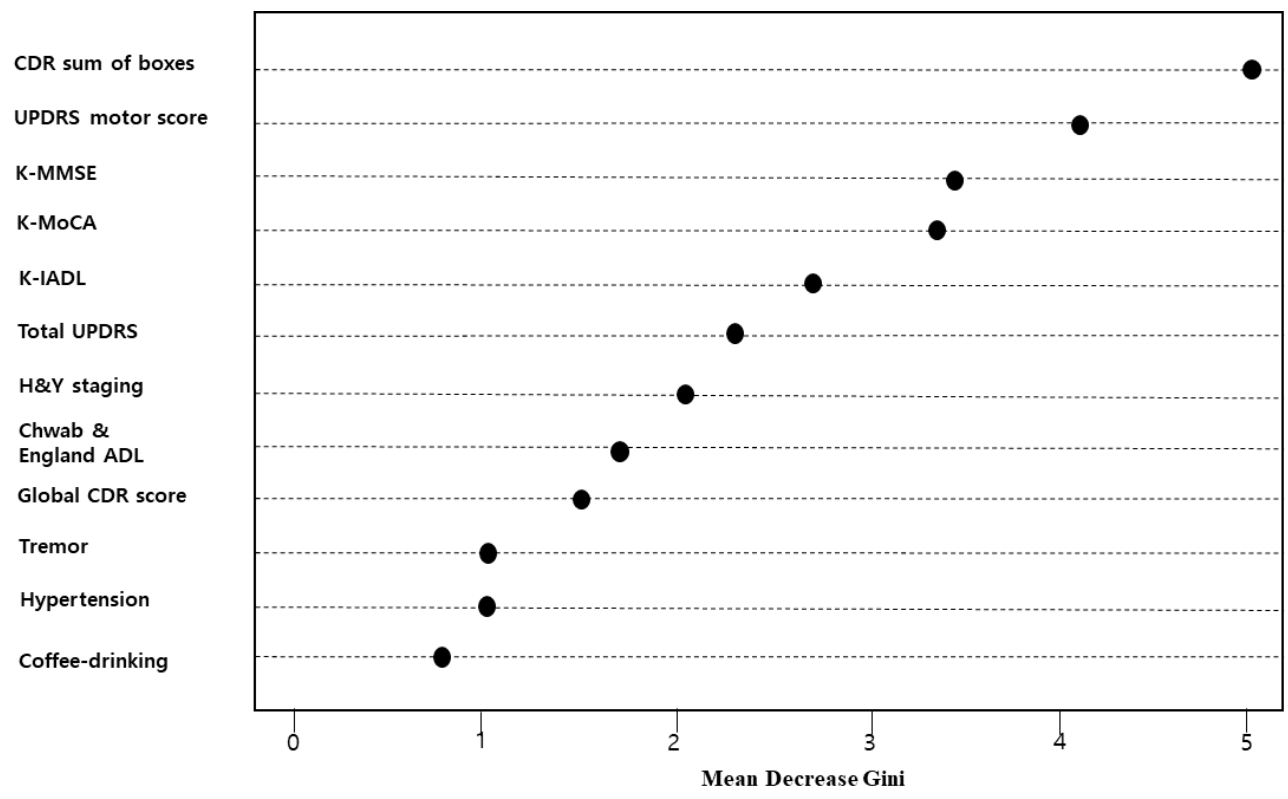

Figure 3. Variable importance in a random forest model (showing only the top 12 factors).

The partial dependence plot regarding the CDR sum of boxes, the most important variable in the predictive model, is presented in Figure 4. The results showed that, when other factors were constant, the risk of PD-MCI increased with a higher CDR sum of boxes (Figure 4).

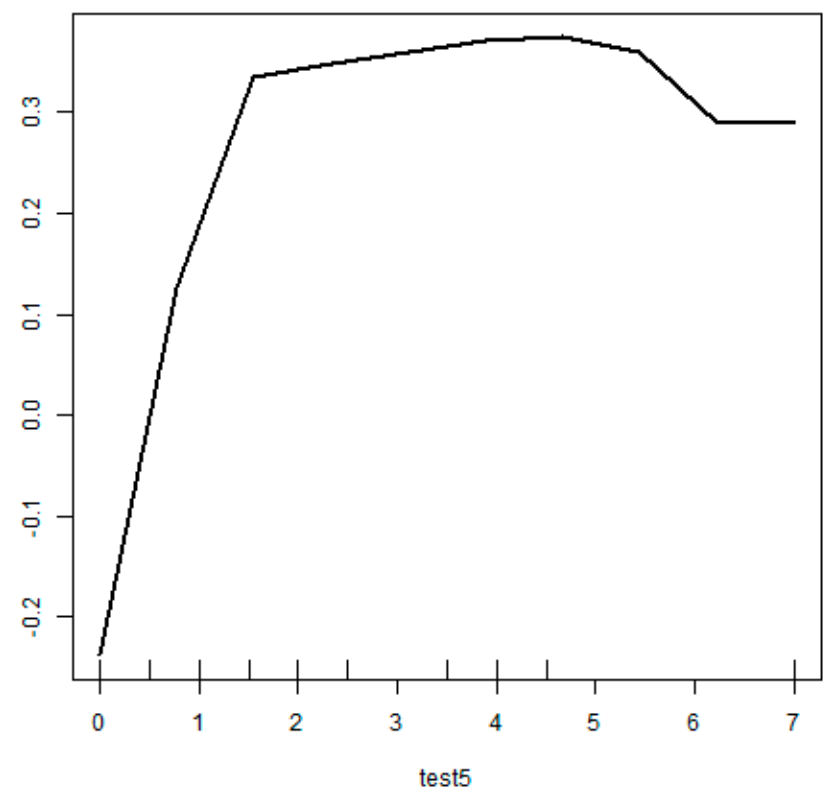

Figure 4. Partial dependence plot (CDR sum of boxes).

\subsection{Comparison of the Accuracy of the Developed Prediction Models}

This study changed the mtry values (numbers), presenting the number of explanatory variables to be used in the decision tree constituting RF, from 5 to 15 , and selected the value with the smallest error of Out-Of-Bag. The changes in the error of Out-Of-Bag are presented in Table 3. The optimal mtry to be applied in this study was 5 , showing the lowest error rate (34.4\%). 
Table 3. Error of out-of-bag.

\begin{tabular}{cc}
\hline Numbers of mtry & Error of Out-of-Bag \\
\hline 5 & 0.344 \\
6 & 0.375 \\
7 & 0.396 \\
8 & 0.375 \\
9 & 0.396 \\
10 & 0.365 \\
11 & 0.385 \\
12 & 0.375 \\
13 & 0.375 \\
14 & 0.375 \\
15 & 0.375 \\
\hline
\end{tabular}

When ntree, the number of tree generation, and mtry were set as 500 and 5, respectively, the final RF model of this study had an overall accuracy of $65.6 \%$, a sensitivity of $70.6 \%$, and a specificity of $60.0 \%$ (Table 4 ). On the other hand, the overall accuracy of CART was calculated as $67.7 \%$, higher than that of RF, but the sensitivity of it was the lowest (51.1\%). In Figure 4, the black line indicates the changes in each error rate against 500 bootstrap samples. Figure 5 shows that the changes in error rate become relatively stable after the number of bootstrap samples exceeded 150 .

Table 4. Comparison of accuracies developed prediction models, \%.

\begin{tabular}{lccc}
\hline \multicolumn{1}{c}{ Model } & Overall Accuracy & Sensitivity & Specificity \\
\hline Multiple logistic & NA & NA & NA \\
regression & 67.7 & 51.1 & 82.4 \\
Decision tree & 65.6 & 70.6 & 60.0 \\
Random Forest & & \\
\hline
\end{tabular}

NA—not available.

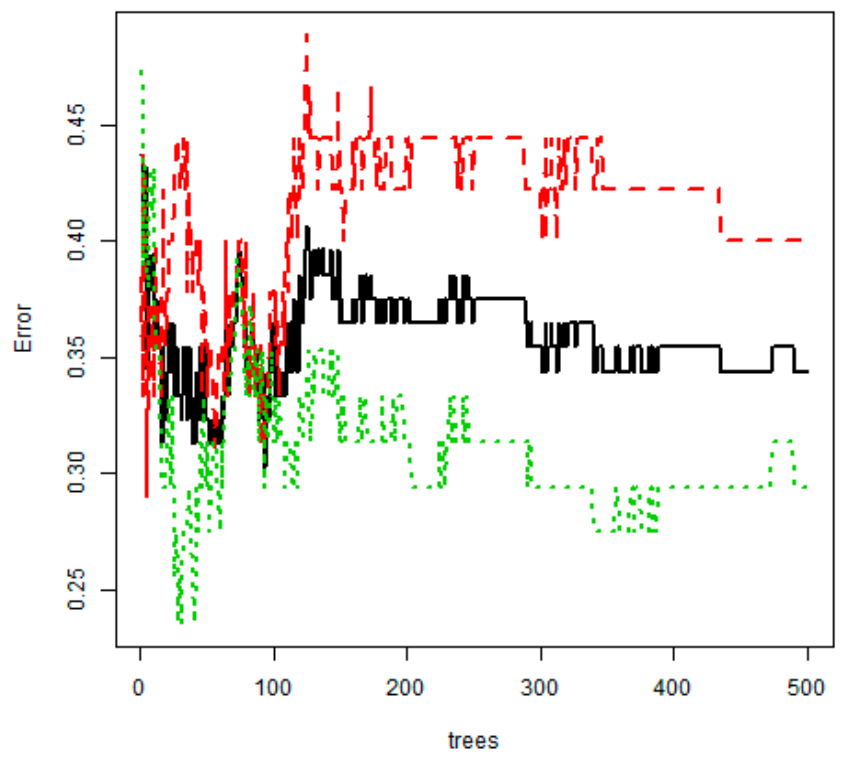

Figure 5. Out-of-bag error rate curve (random forest model). Black line-overall accuracy; red line—sensitivity; Green line-specificity. 


\section{Discussion}

Diagnosing early stage PD-MCI is important in the health sciences, because it can delay the cognitive decline associated with PDD. Previous studies [22,40] have reported that the impairment of the executive function is a major cognitive feature of PDD. However, it is challenging to distinguish PD-MCI from PD-NC solely based on executive function. Therefore, we explored the major differential indicators of PD-MCI, taking into account sociodemographic variables, health habits, PD related motor and non-motor symptoms, cognitive tests, and neuropsychological tests. We developed a PD-MIC prediction model based on random forests, and confirmed that the CDR sum of boxes, UPDRS motor score, K-MMSE total score, and the K-MoCA total score were major predictors of PD-MCI. Among all of the neuropsychological screening tests, the CDR sum of boxes was the most important predictor for distinguishing PD-MCI from PD-NC. Therefore, when a neuropsychological test is performed to diagnose PD-MCI in patients with PD, the CDR (sum of boxes) scoring should be conducted first over other cognitive-language screening tests so as to achieve higher sensitivity.

Previous studies [41,42] examining the sociodemographic and emotional characteristics of PDD reported that depression is the main characteristic of PDD. For example, Aarsland et al. (2007) [41] evaluated 537 patients with PDD and observed that $58 \%$ of the patients had depression. However, in the present study, depression was not an important indicator for predicting PD-MCI. This might differ from previous studies [41,42], because previous studies compared healthy elderly individuals versus those with PD-MCI, while the present study only examined people with PD. In other words, depression is potentially not a major differential indicator in this study, because both PD and PD-MCI have high depression rates $(31.3 \%)$. As only a few studies have tried to distinguish PD-MCI from PD-NC considering neuropsychological characteristics, health habits, and depression, more observation studies on PD-MCI are needed in order to verify the major predictors of PD-MCI.

Another meaningful finding of this study is that the sensitivity of random forests is higher than that of the decision tree model. These results agree with the results of previous studies predicting $\mathrm{MCI}$ [6] or cardiovascular disease in the elderly using random forests [43]. The prediction accuracy of random forests is higher than that of regression models or decision trees, because random forests are based on the bagging algorithm, which generates diverse decision trees using 500 bootstrap samples. As outliers can form decision tree nodes, the effects of the parameters that determine nodes are substantial, and, consequently, carry a risk of overfitting [44]. In contrast, random forests based on the bagging algorithm can prevent overfitting, because they reduce variance while maintaining tree bias. Moreover, random forests achieve a higher prediction accuracy than decision trees [45]. In addition, one advantage of random forests is their reduction of variance compared with the bagging model, which is achieved by decreasing the correlation between trees [43]. Random forests show a particularly better prediction accuracy than bagging models when there are many input variables [43]. Therefore, when selecting the key independent variables from a dataset containing many independent variables, such as the disease data used in this study, or developing prediction models on big data, random forests provide a higher accuracy than decision tree or multiple logistic regression models.

The merit of this study was the development of an MCI prediction model using examination data from a national survey. The limitations of this study are the following: (1) The number of study subjects was small. (2) The obsessive-compulsive symptoms commonly observed in patients with PD were not examined. (3) The prediction model did not include a biomarker, such as CFS. (4) This study adjusted the balance of the number of subjects between the groups by using age-matched PSM to solve the problem of unbalanced data. However, as a result of the PSM, a number of samples were excluded from the analysis, and the same size decreased. As a result, the overall accuracy, sensitivity, and specificity of the multiple logistic regression analysis were not calculated. Moreover, the age used for matching could not be used as an explanatory variable in the predictive model. Future studies will require more advanced techniques that can reduce the probability of overfitting to minimize imbalance, in addition to PSM. (5) Subjects taking PD medications (e.g., dopaminergics) were not 
evaluated. As PD medication particularly affects the expression of cognitive and behavioral symptoms, future studies should consider whether or not a subject takes medication.

\section{Conclusions}

It is necessary to develop a protocol that can easily identify early stage PDD in order to establish individualized monitoring for tracking high-risk groups based on the PD-MCI prediction model developed in this study. Moreover, to further increase the prediction accuracy of the present method, a random forest model using weighted voting is warranted. In addition, the development of multi-modal data-based machine learning models that include biomarkers and brain imaging test indicators, as well as sociodemographic factors, health habits, and neuropsychiatric indicators, is needed.

Funding: This research was supported by the Basic Science Research Program through the National Research Foundation of Korea (NRF) funded by the Ministry of Education (NRF-2018R1D1A1B07041091 and NRF-2019S1A5A8034211).

Conflicts of Interest: The author declares no conflict of interest.

\section{References}

1. Broeders, M.; De Bie, R.M.A.; Velseboer, D.C.; Speelman, J.D.; Muslimovic, D.; Schmand, B. Evolution of mild cognitive impairment in Parkinson disease. Neurology 2013, 81, 346-352. [CrossRef]

2. Goldman, J.G.; Aggarwal, N.T.; Schroeder, C.D. Mild cognitive impairment: An update in Parkinson's disease and lessons learned from Alzheimer's disease. Neurodegener. Dis. Manag. 2015, 5, 425-443. [CrossRef]

3. Ciafone, J.; Little, B.; Thomas, A.J.; Gallagher, P. The Neuropsychological Profile of Mild Cognitive Impairment in Lewy Body Dementias. J. Int. Neuropsychol. Soc. 2020, 26, 210-225. [CrossRef]

4. Nicoletti, A.; Luca, A.; Baschi, R.; Cicero, C.E.; Mostile, G.; Davì, M.; Pilati, L.; Restivo, V.; Zappia, M.; Monastero, R. Incidence of Mild Cognitive Impairment and Dementia in Parkinson's Disease: The Parkinson's Disease Cognitive Impairment Study. Front. Aging. Neurosci. 2019, 11, 21. [CrossRef] [PubMed]

5. Hely, M.A.; Reid, W.G.; Adena, M.A.; Halliday, G.M.; Morris, J.G. The Sydney multicenter study of Parkinson's disease: The inevitability of dementia at 20 years. Mov. Disord. 2008, 23, 837-844. [CrossRef] [PubMed]

6. Byeon, H. A prediction model for mild cognitive impairment using random forests. IJACSA 2015, 6, 8-12. [CrossRef]

7. Geda, Y.E.; Roberts, R.O.; Knopman, D.S.; Petersen, R.C.; Christianson, T.J.; Pankratz, V.S.; Smith, G.E.; Boeve, B.F.; Ivnik, R.J.; Tangalos, E.G.; et al. Prevalence of neuropsychiatric symptoms in mild cognitive impairment and normal cognitive aging: Population-based study. Arch. Gen. Psychiatry 2008, 65, 1193-1198. [CrossRef] [PubMed]

8. Langa, K.M.; Levine, D.A. The diagnosis and management of mild cognitive impairment: A clinical review. JAMA 2014, 312, 2551-2561. [CrossRef]

9. Bott, N.T.; Johnson, E.T.; Schuff, N.; Galifianakis, N.; Subas, T.; Pollock, J.; Pressman, P.; Kramer, J.H.; Possin, K.L. Sensitive measures of executive dysfunction in non-demented Parkinson's disease. Parkinsonism Relat. Disord. 2014, 20, 1430-1433. [CrossRef]

10. Sudo, F.K.; Alves, C.E.O.; Alves, G.S.; Ericeira-Valente, L.; Tiel, C.; Moreira, D.M.; Laks, J.; Engelhardt, E. White matter hyperintensities, executive function and global cognitive performance in vascular mild cognitive impairment. Arq. Neuropsiquiatr. 2013, 71, 431-436. [CrossRef]

11. Petersen, R.C. Early diagnosis of Alzheimer's disease: Is MCI too late? Curr. Alzheimer. Res. 2009, 6, 324-330. [CrossRef] [PubMed]

12. Gabryelewicz, T.; Styczynska, M.; Luczywek, E.; Barczak, A.; Pfeffer, A.; Androsiuk, W.; Chodakowska-Zebrowska, M.; Wasiak, B.;Peplonska, B.; Barcikowska, M. The rate of conversion of mild cognitive impairment to dementia: Predictive role of depression. Int. J. Geriatr. Psychiatry 2007, 22, 563-567. [CrossRef] [PubMed]

13. Lang, M.; Rosselli, M.; Greig, M.T.; Torres, V.L.; Vélez-Uribe, I.; Arruda, F.; Barker, W.W.; Garcia, P.; Loewenstein, D.A.; Curiel, R.E.; et al. Depression and the Diagnosis of MCI in a Culturally Diverse Sample in the United States. Arch. Clin. Neuropsychol. 2019. [CrossRef] [PubMed] 
14. Koh, S.B.; Kwon, D.Y.; Lee, J.M.; Han, J.K.; Kim, B.J.; Park, M.K.; Park, K.W.; Lee, D.H. Prevalence of Parkinsonism in Ansan-city. J. Korean Neurol. Assoc. 2003, 21, 498-501.

15. Kim, H.M.; Nazor, C.; Zabetian, C.P.; Quinn, J.F.; Chung, K.A.; Hiller, A.L.; Shu, CH.; Leverenz, J.B.; Montine, T.J.; Edwards, K.L.; et al. Prediction of cognitive progression in Parkinson's disease using three cognitive screening measures. Clin. Parkinsonism Relat. Disord. 2019, 1,91-97. [CrossRef]

16. Barnish, M.; Daley, D.J.; Deane, K.H.; Clark, A.B.; Gray, R.J.; Horton, S.M.; Butterfint, Z.R.; Myint, P.K. Cognitive profile and determinants of poor cognition in people without dementia in Parkinson's disease. Med. J. Islam. Repub. Iran 2019, 33, 1.

17. Byeon, H. Predicting the Swallow-Related Quality of Life of the Elderly Living in a Local Community Using Support Vector Machine. Int. J. Environ. Res. Public Health 2019, 16, 4269. [CrossRef]

18. Joloudari, J.H.; Hassannataj Joloudari, E.; Saadatfar, H.; GhasemiGol, M.; Razavi, S.M.; Mosavi, A.; Nabipour, N.; Shamshirband, S.; Nadai, L. Coronary Artery Disease Diagnosis; Ranking the Significant Features Using a Random Trees Model. Int. J. Environ. Res. Public Health 2020, 17, 731. [CrossRef]

19. Byeon, H. Development of Depression Prediction Models for Caregivers of Patients with Dementia Using Decision Tree Learning Algorithm. Int. J. Gerontol. 2019, 13, 314-319.

20. Chen, J.; Li, Q.; Wang, H.; Deng, M. A machine learning ensemble approach based on random forest and radial basis function neural network for risk evaluation of regional flood disaster: A case study of the Yangtze River Delta, China. Int. J. Environ. Res. Public Health 2020, 17, 49. [CrossRef]

21. Byeon, H. Developing a random forest classifier for predicting the depression and managing the health of caregivers supporting patients with Alzheimer's Disease. Technol. Health Care 2019, 27, 531-544. [CrossRef] [PubMed]

22. Byeon, H.; Jin, H.; Cho, S. Development of Parkinson's disease dementia prediction model based on verbal memory, visuospatial memory, and executive function. J. Med. Imaging Health Inform. 2017, 7, 1517-1521. [CrossRef]

23. Zhang, J.; Sokal, I.; Peskind, E.R.; Quinn, J.F.; Jankovic, J.; Kenney, C.; Chung, K.A.; Millard, S.P.; Nutt, J.G.; Montine, T.J. CSF multianalyte profile distinguishes Alzheimer and Parkinson diseases. Am. J. Clin. Pathol. 2008, 129, 526-529. [CrossRef] [PubMed]

24. Chaturvedi, M.; Hatz, F.; Gschwandtner, U.; Bogaarts, J.G.; Meyer, A.; Fuhr, P.; Roth, V. Quantitative EEG (QEEG) measures differentiate Parkinson's disease (PD) patients from healthy controls (HC). Front. Aging Neurosci. 2017, 9, 3. [CrossRef] [PubMed]

25. Chaturvedi, M.; Bogaarts, J.; Hatz, F.; Gschwandtner, U.; Cozac, V.; Meyer, A.; Liepelt, I.; Babiloni, C.; Fuhr, P.; Roth, V. Distinguishing Parkinson's Disease Dementia (PDD) patients from Parkinson's Disease (PD) patients using EEG frequency and connectivity measures. Clin. Neurophysiol. 2018, 129, e92. [CrossRef]

26. Lee, J.E.; Kim, J.H.; Hong, E.J.; Yoo, H.S.; Nam, H.Y.; Park, O. National Biobank of Korea: Quality control programs of collected-human biospecimens. Osong. Public Health Res. Perspect. 2012, 3, 185-189. [CrossRef]

27. Winblad, B.; Palmer, K.; Kivipelto, M.; Jelic, V.; Fratiglioni, L.; Wahlund, L.O.; Nordberg, A.; Bäckman, L.; Albert, M.; Almkvist, O.; et al. Mild cognitive impairment-beyond controversies, towards a consensus: Report of the International Working Group on Mild Cognitive Impairment. J. Intern. Med. 2004, 256, 240-246. [CrossRef]

28. Byeon, H. The risk factors of laryngeal pathology in Korean adults using a decision tree model. J. Voice 2015, 29, 59-64. [CrossRef]

29. Ghavami, P. Big Data Analytics Methods: Analytics Techniques in Data Mining, Deep Learning and Natural Language Processing; Walter de Gruyter: Berlin, Germany, 2019.

30. Zhang, Z.; Kim, H.J.; Lonjon, G.; Zhu, Y. Balance diagnostics after propensity score matching. Ann. Transl. Med. 2019, 7, 16. [CrossRef]

31. Kang, Y.; Na, D.L.; Hahn, S. A validity study on the Korean Mini-Mental State Examination (K-MMSE) in dementia patients. J. Korean Neurol. Assoc. 1997, 15, 300.

32. Kang, Y.; Park, J.; Yu, K.H.; Lee, B.C. The validity of the Korean-Montreal Cognitive Assessment (K-MoCA) as a screening test for both MCI and VCI. In Proceedings of the 20th Annual Rotman Research Institute Conference, Toronto, ON, Canada, 22-26 March 2010. [CrossRef]

33. Cho, M.J.; Bae, J.N.; Suh, G.H.; Hahm, B.J.; Kim, J.K.; Lee, D.W.; Kang, M.H. Validation of geriatric depression scale, Korean version (GDS) in the assessment of DSM-III-R major depression. J. Korean Neuropsychiatr. Assoc. $1999,38,48-63$. 
34. Choi, S.H.; Na, D.L.; Lee, B.H.; Hahm, D.S.; Jeong, J.H.; Yoon, S.J.; Yoo, K.H.; Ha, C.K.; Han, I.W. Estimating the validity of the Korean version of expanded clinical dementia rating (CDR) scale. J. Korean Neurol. Assoc. 2001, 19, 585-591.

35. Kang, S.J.; Choi, S.H.; Lee, B.H.; Kwon, J.C.; Na, D.L.; Han, S.H. The reliability and validity of the Korean Instrumental Activities of Daily Living (K-IADL). J. Korean Neurol. Assoc. 2002, 20, 8-14.

36. Movement Disorder Society Task Force on Rating Scales for Parkinson's Disease. The unified Parkinson's disease rating scale (UPDRS): Status and recommendations. Mov. Disord. 2003, 18, 738-750. [CrossRef] [PubMed]

37. Richards, M.; Marder, K.; Cote, L.; Mayeux, R. Interrater reliability of the Unified Parkinson's Disease Rating Scale motor examination. Mov. Disord. 1994, 9, 89-91. [CrossRef] [PubMed]

38. Hoehn, M.M.; Yahr, M.D. Parkinsonism: Onset, progression and mortality. Neurology 1967, 17, 427-442. [CrossRef]

39. Gillingham, F.J.; Donaldson, M.C. Schwab and England Activities of Daily Living. In Third Symposium of Parkinson's Disease; E\&S Livingstone: Edinburgh, UK, 1969.

40. Woods, S.P.; Tröster, A.I. Prodromal frontal/executive dysfunction predicts incident dementia in Parkinson's disease. J. Int. Neuropsychol. Soc. 2003, 9, 17-24. [CrossRef]

41. Aarsland, D.; Brønnick, K.; Ehrt, U.; De Deyn, P.P.; Tekin, S.; Emre, M.; Cummings, J.L. Neuropsychiatric symptoms in patients with Parkinson's disease and dementia: Frequency, profile and associated care giver stress. J. Neurol. Neurosurg. Psychiatry 2007, 78, 36-42. [CrossRef]

42. Prell, T.; Witte, O.W.; Grosskreutz, J. Biomarkers for dementia, fatigue, and depression in Parkinson's disease. Front. Neurol. 2019, 10, 195. [CrossRef]

43. Byeon, H. Developing a model to predict the occurrence of the cardiocerebrovascular disease for the Korean elderly using the random forests algorithm. IJACSA 2018, 9, 494-499.

44. Larose, D.T. Discovering Knowledge in Data: An Introduction to Data Mining; John Wiley \& Sons: Hoboken, NJ, USA, 2014.

45. Lunetta, K.L.; Hayward, L.B.; Segal, J.; Van Eerdewegh, P. Screening large-scale association study data: Exploiting interactions using random forests. BMC Genet. 2004, 5, 32. [CrossRef] [PubMed]

(C) 2020 by the author. Licensee MDPI, Basel, Switzerland. This article is an open access article distributed under the terms and conditions of the Creative Commons Attribution (CC BY) license (http://creativecommons.org/licenses/by/4.0/). 